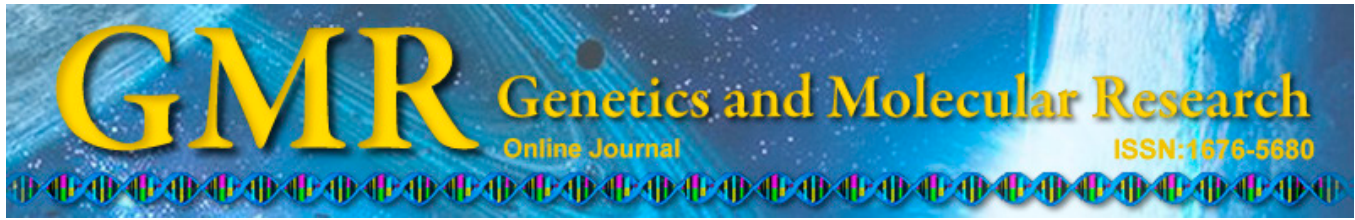

\title{
Therapeutic influence of intraperitoneal injection of Wharton's jelly-derived mesenchymal stem cells on oviduct function and fertility in rats with acute and chronic salpingitis
}

\author{
H.J. Luo ${ }^{1}$, X.M. Xiao ${ }^{1}$, J. Zhou ${ }^{1}$ and W. Wei ${ }^{2}$ \\ ${ }^{1}$ Obstetrics and Gynecology Department, The First Affiliated Hospital \\ of Jinan University, Huangpu Road West, Tianhe District, Guangzhou, \\ Guangdong Province, China \\ ${ }^{2}$ The Cord Blood Bank of Guangdong Province of China, Guangzhou, \\ Guangdong Province, China
}

Corresponding author: X.M. Xiao

E-mail: luohuijuan_1hj@163.com

Genet. Mol. Res. 14 (2): 3606-3617 (2015)

Received August 26, 2014

Accepted December 3, 2014

Published April 17, 2015

DOI http://dx.doi.org/10.4238/2015.April.17.10

\begin{abstract}
To study the effect of Wharton's jelly-derived mesenchymal stem cells (WJMSCs) on the rat salpingitis model, 50 female Wistar rats were randomly divided into one control and five model groups. Mixed bacteria were injected into the oviducts of model groups. The treated acute and chronic groups received intraperitoneal injections of WJMSCs $\left(\begin{array}{lll}1 & \mathrm{x} & 0^{6}\end{array}\right)$ once a week for three weeks. Serum inflammation factor, collagen fiber content and oviduct-specific glycoprotein (OVGP) levels were detected in control, chronic, ex-treatment acute and chronic, and treated acute and chronic groups ( $\mathrm{N}=5$ for each group). Pregnancy rate and litter size of control, chronic, treated acute and treated chronic groups were compared. Serum TNF- $\alpha$ and INF- $\gamma$ levels increased in ex-treatment
\end{abstract}


acute and chronic groups, and restored to normal in the acute treated group but not in the treated chronic group. Oviduct collagen fibers did not increase significantly before or after treatment in the acute group, but it increased in the ex-treatment chronic group and did not improve after treatment. After treatment, OVGP levels restored to normal in the acute group but reduced in the ex-treatment and treated chronic groups and chronic group. The pregnancy rate and litter size of the treated acute group recovered to normal, but in the treated chronic group and chronic model group, they decreased significantly. Thus, intraperitoneal injection of WJMSCs could recover the function of the oviduct in acute salpingitis rats, but its effect on chronic salpingitis was poor. Timely treatment of salpingitis is crucial to preserve reproductive function.

Key words: Wharton's jelly-derived mesenchymal stem cells; Rat; Salpingitis; Collagen Fiber; Oviduct-specific Glycoprotein

\section{INTRODUCTION}

Among women of reproductive age worldwide, the infertility rate is as high as 10$15 \%$ (Abeles et al., 2013), among which, 30-35\% is caused by oviduct infertility (Araki et al., 2003). Chronic tubal inflammatory injury is the main cause of oviduct infertility. In recent years, due to the popularity of sexually transmitted disease, its incidence has presented a rising trend.

Wharton's jelly-derived mesenchymal stem cells (WJMSCs) can differentiate into cartilage cells, hair follicle epithelial cells, liver cells, female reproductive cells or neural cells under specified conditions, showing multidirectional differentiation potential and acting as seed cells for cell replacement therapy (Buhi, 2002; Breymann et al., 2006; Chang et al., 2011; Chen et al., 2012). They do not express Main histocompatibility complex (MHC) II and have low immunogenicity and low or no expression of MHCI (Gnoth et al., 2005; Cohen and Prince, 2013); hence, they have been widely used in fields such as genetics, immunology, and tissue repair and are the hot topic in stem cell research. Treatment of infertile patients with oviduct inflammatory injury using WJMSCs to restore the normal structure and function of the oviduct and to cure oviduct infertility has not yet been reported. Because WJMSCs are easily obtained in clinical obstetrics and possess pluripotent stem cell potential, high differentiation capacity, anti-inflammatory, anti-fibrosis and homing functions, further research, both in vivo and in vitro, is definitely worthwhile.

This study adopted the "mixed bacteria" method to establish a salpingitis rat model (acute and chronic), treated the model with intraperitoneal injections of WJMSCs, and compared serum TNF- $\alpha$ and IFN- $\gamma$ concentrations, morphological changes of oviduct under light microscope, changes of tubal peristalsis and secretion function, and pregnancy rates between groups to determine the curative effects of WJMSCs on acute and chronic salpingitis rat models. Here we discuss the working mechanism and predict its clinical application prospects. 


\section{MATERIAL AND METHODS}

\section{Materials and animals}

\section{Wharton's jelly-derived mesenchymal stem cells}

The umbilical cord tissue of healthy and full term infants, who were born through cesarean delivery due to non-pathological factors, was taken for preparation. HBsAg, anti-HCV, anti-HIV, CMV-IgM, mycoplasma, chlamydia, and syphilis tests were all negative. Tissue block was adherent to pot wall for culture. Following culture for three to seven days, fusiform fibroblasts began growing around the culture dish walls and expanded up to $80 \%$ of the culture dish surface in approximately two weeks. Flow cytometry was used to detect cell expression markers: (positive CD29, CD44, CD71, CD73, CD90, CD105, and negative CD14, CD34, CD45, CD117) to determine the cells to be WJMSCs. The cell vitality was $95-97 \%$ and solutions were diluted with saline water to the concentration of $1 \times 10^{6}$ cells $/ \mathrm{mL}$. All were made and provided by the Cord Blood Bank of Guangdong Province of China on the day of use. This study was approved by the Institutional Review Board of The First Clinical Medical College of JINAN University within which the study was undertaken.

\section{Model-making bacterial strain}

Bacterial suspensions with a concentration of $3 \times 10^{9}$ cells $/ \mathrm{mL}$ were prepared by mixing Escherichia coli (ATCC25922), Staphylococcus aureus (ATCC25923) and hemolytic Streptococcus (ATCC21059) at a ratio of 2:1:1. All were provided by the Department of Microbiology of Jinan University's medical faculty.

\section{Experimental animals}

SPF Wistar female rats $(\mathrm{N}=50)$ and 20 male rats, aged six-eight weeks (weighing 200 $\pm 20 \mathrm{~g}$ ) were provided by the Animal Experiment Center of Guangzhou University of Chinese Medicine (qualification No. 2005A028). The animal experimental protocol was approved by the Institutional Animal Research Ethics Review Board. Animals were used in accordance with the Animal Care and Use Committee of JINAN University.

\section{Main experimental reagents}

Quick EIA ${ }^{\mathrm{TM}}$ rat IFN- $\gamma / \mathrm{TNF}-\alpha$ ELISA kit: Shenzhen Dakewe Biological Technology Co., LTD. Rabbit anti-rat oviduct-specific glycoprotein (OVGP)-Ab: ABCON, the United States. Biotinylated goat anti rabbit IgG antibody -HRP polymer: Beijing Sequoia Jinqiao Biotechnology Co., Ltd.

\section{Researched animal grouping}

Wistar female rats $(\mathrm{N}=50)$ were randomly divided into a control group of 10 rats and model groups. It had been determined by preliminary experiments that the 20th model day was 
the peak of acute inflammation and the 30th day was representative of chronic inflammation of salpingitis.

After anesthesia by pentobarbital sodium, hair removal and disinfection techniques were applied to rats' bellies. The abdominal cavity was opened and bilateral oviducts were found along the horn of uterus. Right at the uterine horn-tubal junction, $0.05 \mathrm{~mL}$ of mixed bacteria was injected into bilateral oviducts of model rats, which were further divided randomly into five groups. While $0.05 \mathrm{~mL}$ saline was injected into rats of the control group. The rats were divided as follows: 1) Control group: five of 10 rats were randomly selected on the 51st day of the experiment to take 5-6 $\mathrm{mL}$ of blood through the abdominal aorta and centrifuge for five min at a speed of $3616 \mathrm{~g}$ (Eppendorf 5418) to isolate the serum for later use. The right oviduct was extracted, fixed with $4 \%$ paraformaldehyde, made into paraffin slices and observed by H\&E staining while the remaining five rats were caged together with Wistar male rats (1:1) for 30 days. 2) Ex-treatment acute group: five model rats were euthanized 20 days after bacteria injection. 3) Treated acute group: from the 20th modeling day, 10 model rats received intraperitoneal injection of $1 \times 10^{6} / \mathrm{mL}$ WJMSCs each at three different times (at seven day intervals), five of which were randomly selected and euthanized on the 41st day (that is, one week after the treatment) and the remaining five were caged with male rats for 30 days. 4) Ex-treatment chronic group: five model rats were euthanized 30 days after injection with bacteria. 5) Treated chronic group: 10 model rats were given the same treatment as group three, from the 30th day after bacteria injection and then five were randomly selected and euthanized one week after treatment (on the 51st day after bacteria injection) and the remaining five were caged with male rats for 30 days. 6) Chronic model group: 10 rats were given no intraperitoneal injection of $1 \times 10^{6} / \mathrm{mL}$ WJMSCs and then five were randomly selected and euthanized on the 51st day after bacteria injection and the remaining five were caged with male rats for 30 days.

\section{Experimental procedures}

\section{ELISA testing procedures}

After dilution, different concentrations of cytokines were put into the standard hole and $50 \mu \mathrm{L}$ of sample was added to each sample hole. A blank hole was also set up. After adding diluted biotin labeling rat serum TNF- $\alpha$ and IFN- $\gamma$ antibodies $(50 \mu \mathrm{L})$ to the blend, samples were incubated at $37^{\circ} \mathrm{C}$ for $1.5 \mathrm{~h}$. We added diluted streptavidin-HRP and incubated at $37^{\circ} \mathrm{C}$ for $30 \mathrm{~min}$. Then, we incubated with $\mathrm{TMB}$ for $30 \mathrm{~min}$ at $37^{\circ} \mathrm{C}$ (avoiding light) and then quickly added the stop buffer. Within $10 \mathrm{~min}$, a detection wavelength of $450 \mathrm{~nm}$ was used to inspect the OD value. The corresponding concentration of each sample could be counted.

\section{OVGP testing procedure}

Wax sections of each specimen were dewaxed. A drop of $3 \% \mathrm{H}_{2} \mathrm{O}_{2}$ was added to each slice and incubated for 5-10 min at room temperature. A drop of primary antibody (rabbit anti-rat OVGP - Ab, diluted at 1:150) was added to each slice and incubated overnight at $4{ }^{\circ} \mathrm{C}$ (replaced primary antibody with PBS for the negative control). After washing with PBS, we added one drop of biotinylated secondary antibody and incubated each slice for $30 \mathrm{~min}$ at room temperature. After washing, we added one drop of freshly prepared DAB TMB substrate (diaminobenzidine) to each section. Sections were washed fully and redyed with haematin. 


\section{Judgment of results}

Image Pro Plus 6.0 - Image analysis system was used to test the IOD value with tan dyeing depth ranging from light yellow to deep yellow. Views $(N=5)$ were randomly selected from each slice that contained the tubal epithelium with a magnification factor of 200X.

A paraffin slice was cut from each sample, stained with Regaud hematoxylin-Masson ponceaux and observed under the microscope to test the dyeing effects: collagen fibers in blue; cytoplasm, muscle and cellulose in red; and nucleus in black and blue. Five microscopy images from mucous membranes of oviducts were randomly picked up and the image was magnified (200X) to detect the grey level (GL) of blue metachromatic granules.

\section{Statistical method}

The SPSS13.0 statistics software was used for data statistics and measurement data are reported as means \pm standard error (means $\pm \mathrm{SE}$ ). The measurement data of IOD values were too large so IOD values used in this article were expressed in the form of $\mathrm{N} \mathrm{x} 10^{4}$. The chi-squared test was used to express the comparison between groups and the $t$-tests was used to express comparison within groups. $\mathrm{P}<0.05$ was considered to be statistically significant.

\section{RESULTS}

\section{All model rats survived}

The abdominal wounds of the treated acute group and treated chronic group healed and the catgut absorbed completely after treatment with WJMSCs. In chronic model group that was not treated, neck abscess was observed in one rat and catgut in the wound was not absorbed with a little pus scab in two rats on the 51st day of the experiment.

\section{Oviduct morphological change}

Normal rats have rich oviduct mucosa folds, which are covered with single columnar epithelium, and the proportion of submucosa and muscularis is about 1:1. On the 20th day, model rat uterine horn, tubal edema, and empyema were obvious and even abscesses had formed. Blood capillary expansion of the tube wall, massive neutrophil infiltration, mucosal fold disappearing, and epithelial necrosis could be seen. After the three intraperitoneal injections with WJMSCs, oviduct appearance returned back to normal and soft, mucosal folds recovered with the proportion of submucosa and muscularis close to $1: 1$. On the 30th day from bacterial injection, oviduct swelling or enlargement appeared, as well as stenosis or incomplete obstruction in some tubes. Mucosa epithelium was replaced by single squamous epithelium. After therapy, no obvious improvement was observed (Figure 1A-F).

\section{Comparison of collagen fiber staining}

Normal submucosal collagen fibers were bright blue, thin and sparse, with lots of mesenchymal cells within them. Tube wall dyeing was thin due to edema in ex-treated acute groups with less collagen fibers. Edema disappeared after treatment (the collagen fiber dyeing 
showed no statistical difference; $\mathrm{P}>0.05$ ). The ex-treated chronic group had massive granulation tissue, deep collagen staining deep and unclear boundaries. GL values were decreased and the differences were statistically significant compared with both the control group and the treated acute group $(\mathrm{P}<0.05)$. After treatment, submucosal collagen was not reduced. A large number of corrugated, cord-like, dark gray, blue collagen fibers could be seen in the chronic model group (Figure 2A-F). GL values were the lowest in this group and the differences were statistically significant compared with the control group and treated chronic group $(\mathrm{P}<0.05)$. The results are shown in Table 1.
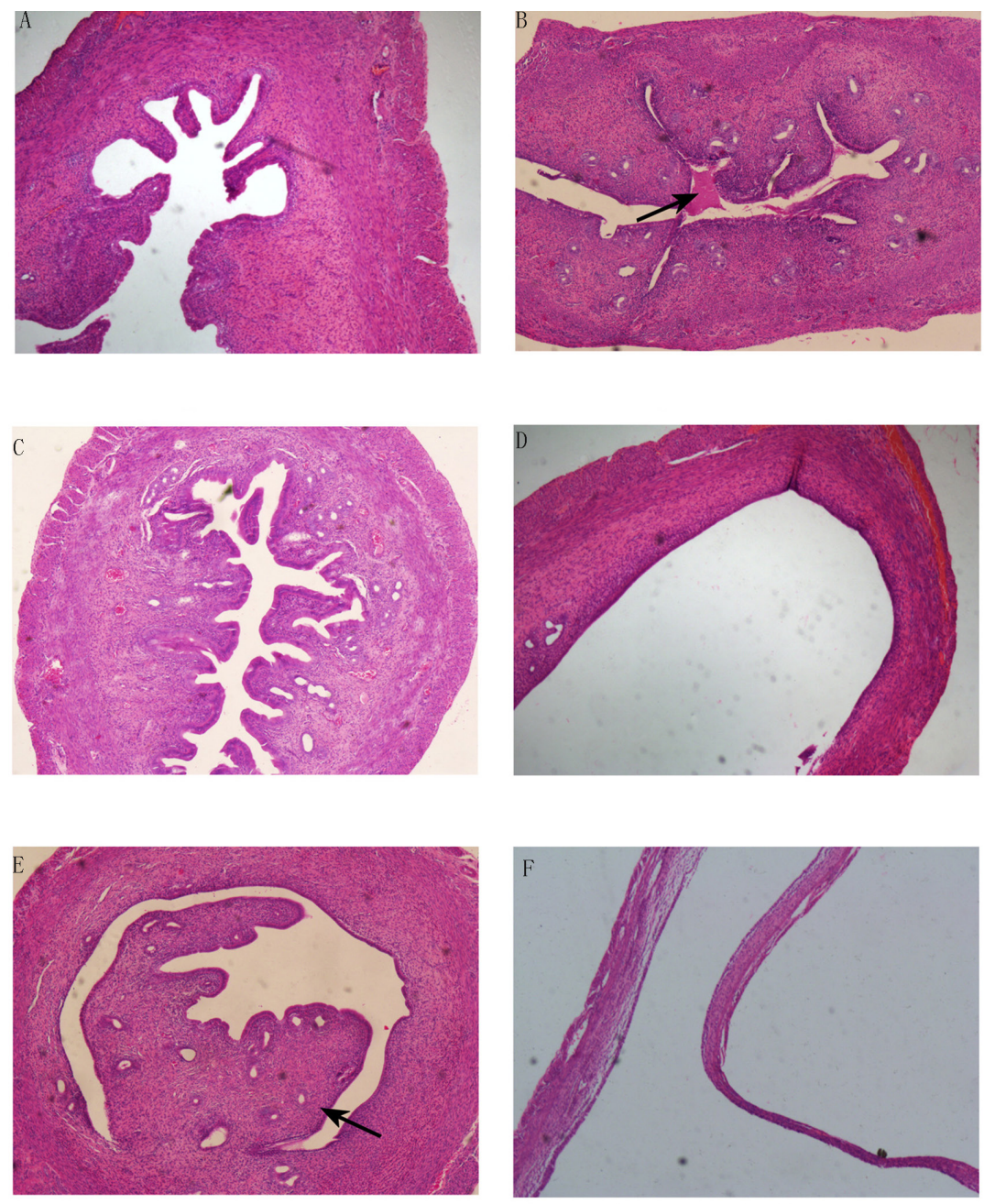

Figure 1. A. Control group: At junction oviduct rich in folds mucosal layer: muscular layer 1:1. B. Ex-treated acute group: Submucosal edema, tube cavity necrosis exudation and epithelial shedding (black arrow). C. Treated acute group: mucosal folds recovered and the proportion of submucosa and muscularis was close to 1:1. D. Extreated chronic group: oviduct swelling and enlargement appeared, mucosa epithelium replaced by single squamous epithelium. E. Treated chronic group: lumen stenosis, polyps and incomplete obstruction (black arrow). F. Chronic model group: serious hydrosalpinx, tubal wall fibrosis (magnified 100X). 

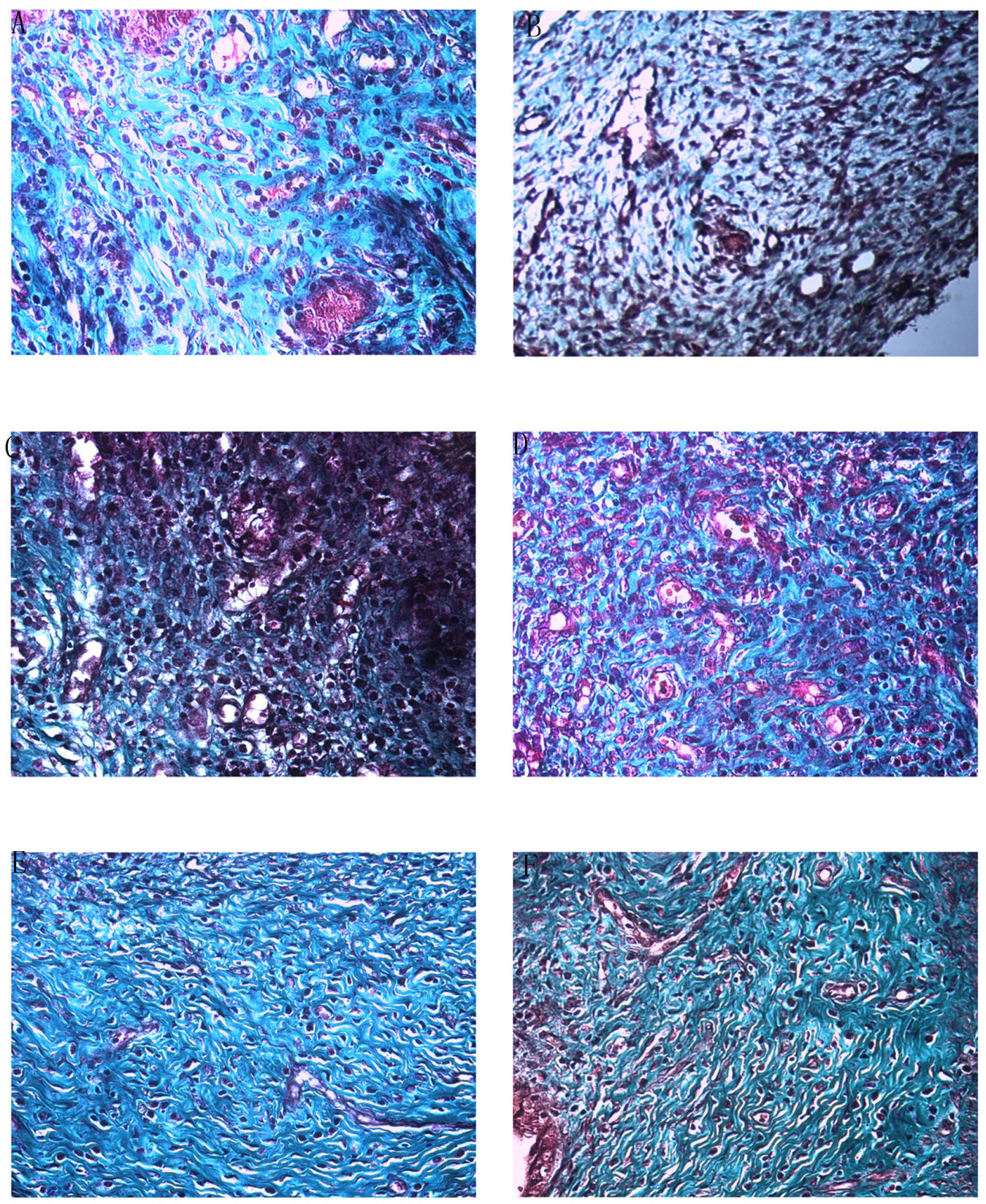

Figure 2. A. Control group: bright blue collagen, thin as cloudiness and collagen outline is not clear. B. Extreated acute groups: tube wall dyeing was thin due to edema. C. Treated acute groups: edema disappeared, with lots of mesenchymal cells within them. D. Ex-treated chronic group: had massive granulation tissue, collagen staining deep. E. Treated chronic group: several corrugated cord collagen fibers were formed and, mesenchymal cells reduced. F. Chronic model group: dying of corrugated cord-like collagen fibers made further deep, dark gray blue (magnified 200X). 


\begin{tabular}{|c|c|c|c|}
\hline Grouping & $\mathrm{N}$ & GL & $\operatorname{OVGP}\left(\mathrm{x} 10^{4}\right)$ \\
\hline Control group & 5 & $92.37 \pm 12.26$ & $4.40 \pm 3.84$ \\
\hline Ex-treated acute group & 5 & $102.46 \pm 36.2^{\mathrm{b}}$ & $5.92 \pm 3.74$ \\
\hline Treated acute group & 5 & $96.00 \pm 28.09^{\mathrm{bc}}$ & $3.15 \pm 2.20$ \\
\hline Ex-treated chronic group & 5 & $70.90 \pm 8.06^{\mathrm{ab}}$ & $1.77 \pm 1.55$ \\
\hline Treated chronic group & 5 & $74.69 \pm 7.00^{\mathrm{ab}}$ & $0.41 \pm 0.54$ \\
\hline Chronic model group & 5 & $60.21 \pm 8.69^{\mathrm{a}}$ & $0.37 \pm 0.22$ \\
\hline F & 2.503 & 9.327 & \\
\hline$P$ & 0.049 & 0.000 & \\
\hline
\end{tabular}

\section{IOD values of OVGP}

Tubal epithelium in the control group had larger and more dark-brown OVGP positive granules gathered in the top pulp part. The tubal epithelium in the ex-treatment acute group was damaged and ruptured with many OVGP-positive particles running into the lumen. Oviduct epithelium in the treated acute group was repaired and many dark brown particles reappeared in the cytoplasm, which was not statistically significant compared with the control group. The ex-treatment chronic group showed squamous metaplasia and the number of OVGP-positive particles decreased significantly as compared with control and acute groups (both before and after treatment) and did not increase significantly after treatment (Figure 3A-F). Tubal epithelium was very thin (single-layer flat) and intraepithelial OVGP-positive particles were rare in the chronic model group. The comparison results of the average optical density of OVGP in each group are shown in Table 1.

\section{Comparison of serum TNF- $\alpha$ and IFN- $\gamma$ concentration between groups}

Serum TNF- $\alpha$ and IFN- $\gamma$ concentration increased significantly in ex-treated acute and chronic groups, which was statistically different from the control group $(\mathrm{P}<0.05)$. However, in the treated acute group, serum TNF- $\alpha$ and IFN- $\gamma$ concentration decreased and returned levels to normal, which were not significantly different from the control group ( $\mathrm{P}>$ $0.05)$. Although serum TNF- $\alpha$ and IFN- $\gamma$ concentrations in the treated chronic group decreased after treatment, they were not statistically different from those before treatment $(\mathrm{P}>0.05)$. In the ex-treated chronic group, TNF- $\alpha$ concentration was statistically different compared with that of the treated acute group $(\mathrm{P}<0.05)$. Serum TNF- $\alpha$ and IFN- $\gamma$ concentrations in the chronic model group were obviously higher than that of the control group, but were not significant compared with those of the treated chronic group $(\mathrm{P}>0.05)$. The results are shown in Table 2.

\section{Comparison of pregnancy rate and litter size between groups of rats}

The five rats that were caged together (with males) in the control and treated acute groups were all pregnant, having a conception rate of $100 \%$ and a litter size of $10 \pm 2.74$ and $11 \pm 3.87$ per mother rat. These were not statistically significantly different from each other $(\mathrm{P}>0.05)$. One of five rats caged together in the treated chronic group gave birth, giving a 
conception rate of $20 \%$ with the average litter size of $2.2 \pm 4.92$. Two rats in the chronic model group gave birth, with the average litter size of $2.2 \pm 4.83$. Those two groups were statistically different from the control group $(\mathrm{P}<0.05)$. The results are shown in Table 3 .
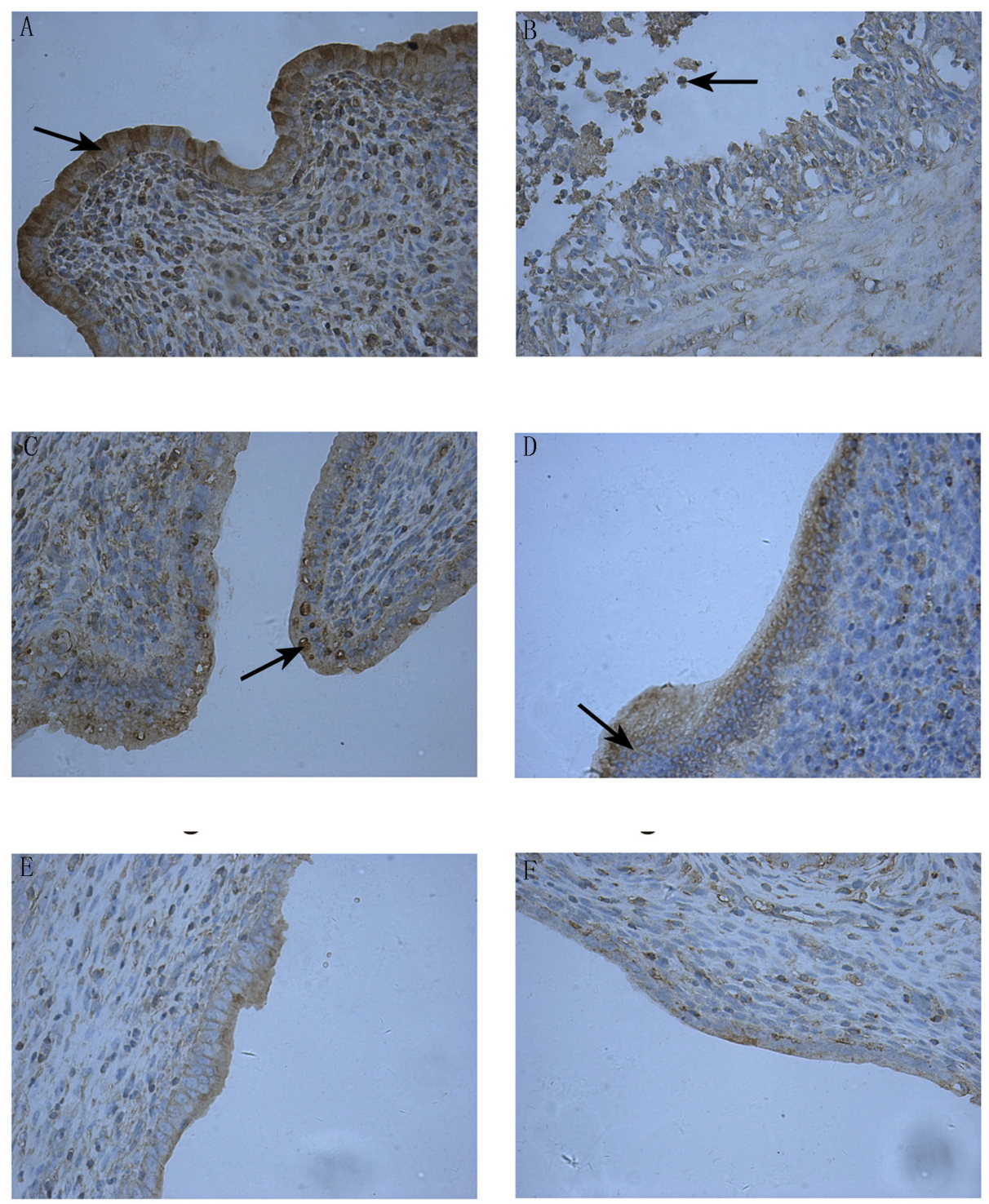

Figure 3. A. Control group: larger and more dark-brown OVGP positive granules gathering in the top pulp part (black arrow). B. Ex-treated acute group: several OVGP-positive particles were released into the oviduct lumen along with cytoplasm (black arrow). C. Treated acute group: tubal epithelium was repaired, dark brown granules were observed in the cytoplasm once more (black arrow). D. Ex-treated chronic group: tubal epithelium had squamous metaplasia (black arrow), OVGP-positive particles decreased significantly. E. Treated chronic group: OVGP-positive particles decreased significantly. F. Chronic model group: mucosa epithelium were replaced by single squamous epithelium and OVGP-positive particles rarely seen (magnified 200X). 
Table 2. Comparison of serum TNF- $\alpha$ and IFN- $\gamma$ concentration among groups.

\begin{tabular}{llcr}
\hline Grouping & $\mathrm{N}$ & TNF- $\alpha(\mathrm{pg} / \mathrm{mL})$ & IFN- $\gamma(\mathrm{pg} / \mathrm{mL})$ \\
\hline Control group & 5 & $575.38 \pm 306.40$ & $706.53 \pm 238.41$ \\
Ex-treated acute group & 5 & $1358.75 \pm 134.05^{\mathrm{ab}}$ & $1280.38 \pm 240.69^{\mathrm{a}}$ \\
Treated acute group & 5 & $579.44 \pm 47.44^{\mathrm{c}}$ & $843.29 \pm 312.42^{\mathrm{b}}$ \\
Ex-treated chronic group & 5 & $1335.33 \pm 60.35^{\mathrm{a}}$ & $1041.02 \pm 146.25^{\mathrm{ab}}$ \\
Treated chronic group & 5 & $1001.74 \pm 168.68$ & $894.14 \pm 767.43$ \\
Chronic model group & 5 & $1477.49 \pm 221.49^{\mathrm{a}}$ & $1296.81 \pm 73.64^{\mathrm{a}}$ \\
\hline
\end{tabular}

$\mathrm{P}<0.05$ means there is statistical difference; $\mathrm{a}$ is the comparison with normal control group; $\mathrm{b}$ is the comparison with chronic model group; $\mathrm{c}$ is the comparison between treated acute group and ex-treated chronic group.

Table 3. Comparison (CP) of pregnancy rate (PR) and litter size (LS) among groups.

\begin{tabular}{|c|c|c|c|c|c|c|}
\hline \multirow[t]{2}{*}{ Grouping } & \multirow[t]{2}{*}{ PR } & \multirow[t]{2}{*}{ LS } & \multicolumn{2}{|c|}{ CP with CG } & \multicolumn{2}{|c|}{ P with $\mathrm{CMG}$} \\
\hline & & & $t^{1}$ & $\mathrm{P}^{1}$ & $t^{2}$ & $\mathrm{P}^{2}$ \\
\hline Control group (CG) & $100 \%$ & $10 \pm 2.74$ & & & & \\
\hline Chronic model group (CMG) & $40 \%$ & $2 \pm 2.83$ & 4.544 & 0.002 & & \\
\hline Treated acute group & $100 \%$ & $11 \pm 3.87$ & 0.471 & 0.650 & 4.196 & 0.003 \\
\hline Treated chronic group & $20 \%$ & $2.2 \pm 4.92$ & 3.098 & 0.015 & 0.079 & 0.939 \\
\hline
\end{tabular}

$\mathrm{P}<0.05$ means there is statistical difference.

\section{DISCUSSION}

Fallopian tubes are a very important part in the reproductive process. At the time of bacterial infection, T-helper lymphocytes and NK cells produce a variety of inflammatory factors, such as TNF- $\alpha$, IFN- $\gamma$, IL-1, 6, 12, 10 and inflammatory cells are recruited to remove bacteria and induce local acute inflammation (Killian, 2011). Columnar epithelium in chronic inflammation was turned into simple squamous epithelium. Stratified squamous epithelium is of stronger resistance (due to inflammation damage and many growth factors) and participates in stimulating hyperplasia of mesenchymal and parenchymal cells and formation of granulation tissue.

\section{Fibrosis of fallopian tube wall}

In chronic inflammation, a large number of inflammatory mediators such as IFN- $\gamma$, TNF- $\alpha$, and IL - 2, 6, 12 can stimulate proliferation of mast cells and fibroblasts to produce large amounts of collagen and induce chronic inflammatory tissue fibrosis gradually causing tissue elasticity and peristalsis to disappear.

The experiment found that tissue edema collagen content was reduced in the ex-treated acute group. After treatment, edema disappeared, the mucosal epithelium regenerated and no obvious fibrosis was seen. In the chronic group, there was massive granulation formed in the tube wall. The collagen did not decrease obviously in the treated chronic group, and in the chronic model group it was the highest. WJMSCs seem to slow down the progress of fibrosis. It is possible that WJMSCs might inhibit the growth of new granulation tissue by inhibiting inflammatory factors (Lee et al., 2013), but it could not dissolve or absorb already formed granulation tissue. 


\section{Oviduct secretion function}

OVGP are multi-protein complexes produced by secretory cells of the oviduct epithelium. Researchers have different opinions about its importance. Whatever its function, the amount of OVGP secretion will reflect the function of endocrine cells.

This experiment showed that tubal epithelium had a very high OVGP level in the control group. Due to cell rupture in the acute inflammation period, a large amount of OVGP was released into the lumen. Thus, OVGP levels decreased in epithelial cells but were high in the lumen. In the treated acute group, columnar epithelial was repaired and secretory function recovered. OVGP expression decreased significantly in the ex-treatment chronic group, treated chronic group and chronic model group, which indicates that the secretory function of tubal epithelium was seriously damaged. It is worth noting that in the treated acute group, intraperitoneal injection of WJMSCs was administered during the acute inflammation period, which made OVGP secretory levels significantly higher than in the ex-treated chronic group. Therefore timely treatment is the key to saving oviduct secretion function.

\section{Influence on systemic inflammation factors}

TNF- $\alpha$ is a multifunctional cell factor produced by mononuclear macrophages and is closely related to reproductive immunization. Produced by activated T cells, IFN- $\gamma$ is the most important cytokine in delayed hypersensitivity skin reaction (DHSR). Both can aggravate oviduct injury, stimulate fibroblast proliferation, participate in inflammatory scar formation and, at the same time, affect the quality of gametes and embryos (Ortiz et al., 2003).

The results show that TNF- $\alpha$ and IFN- $\gamma$ levels in the ex-treated acute and chronic groups increased significantly. They both returned to normal in the treated acute group, which showed that WJMSCs had beneficial effects on acute salpingitis. In the ex-treated chronic group, they both were lower as compared with the treated acute group. This indicates that using WJMSC treatment as soon as possible during an acute infection period could control the progress of inflammation. TNF- $\alpha$ and IFN- $\gamma$ levels, in the chronic model group were obviously higher than in the control group, but there was no significant difference when compared with the treated chronic group. Considering the fact that it is difficult for WJMSCs to go into the oviduct lumen through the occlusive fallopian tube, the residual bacteria within the tubal fluid were not cleared away.

\section{Influence of WJMSC treatment in acute or chronic salpingitis on pregnancy rates}

This experiment confirmed that the treatment of WJMSCs on acute salpingitis can heal and restore tubal reproductive function. The conception rate and litter size of the chronic group showed no significant improvement compared with the control group, which reminded us that repair function of WJMSCs on chronic damage, was limited. It was too late to use WJMSCs to restore reproductive function when tubal function had been severely damaged and fibrosis initiated. Our preliminary experiments had found that when cultured with primitive oviduct epithelial cells in vitro, WJMSCs had the tendency to differentiate as oviduct epithelial cells (Zhou, 2012). Therefore, WJMSCs themselves might be involved in the repair of acute salpingitis injury. However, in this experiment we did not observe obvious anti-fibrosis function of 
WJMSCs treatment on chronic salpingitis. Changing WJMSCs usage to observe its curative effects on chronic salpingitis will be the subsequent research directions of this study.

\section{ACKNOWLEDGMENTS}

This research is part of the larger project entitled "Study On Treatment of Wharton's Jelly-derived Mesenchymal Stem Cells On Tubal Infertility" which was supported by National Natural Science Foundation grants \#81370676 and \#2012B32000007 from Department of Science and Technology of Guangdong Province, China, and grant \#7411785665675 from Department of Science and Technology of Guangzhou city, Guangdong Province, China.

\section{REFERENCES}

Abeles SR, Chuquiyauri R, Tong C and Vinetz JM (2013). Human host-derived cytokines associated with Plasmodium vivax transmission from acute malaria patients to Anopheles darlingi mosquitoes in the Peruvian Amazon. Am. J. Trop. Med. Hyg. 88: 1130-1137.

Araki Y, Nohara M, Yoshida-Komiya H, Kuramochi T, et al. (2003). Effect of a null mutation of the oviduct-specific glycoprotein gene on mouse fertilization. Biochem. J. 374: 551-557.

Breymann C, Schmidt D and Hoerstrup SP (2006). Umbilical cord cells as a source of cardiovascular tissue engineering. Stem. Cell Rev. 2: 87-92.

Buhi WC (2002). Characterization and biological roles of oviduct-specific, oestrogen-dependent glycoprotein. Reproduction 123: 355-362.

Chang JW, Hung SP, Wu HH, Wu WM, et al. (2011). Therapeutic effects of umbilical cord blood-derived mesenchymal stem cell transplantation in experimental lupus nephritis. Cell Transplant. 20: 245-257.

Chen W, Li M, Li Z, Yan Z, et al. (2012). CXCR4-transduced mesenchymal stem cells protect mice against graft-versushost disease. Immunol. Lett. 143: 161-169.

Cohen TS and Prince AS (2013). Activation of inflammasome signaling mediates pathology of acute P. aeruginosa pneumonia. J. Clin. Invest. 123: 1630-1637.

Gnoth C, Godehardt E, Frank-Herrmann P, Friol K, et al. (2005). Definition and prevalence of subfertility and infertility. Hum. Reprod. 20: 1144-1147.

Killian G (2011). Physiology and endocrinology symposium: evidence that oviduct secretions influence sperm function: a retrospective view for livestock. J. Anim. Sci. 89: 1315-1322.

Lee HW, Cheng J, Kovbasnjuk O, Donowitz M, et al. (2013). Insulin-like growth factor 1 (IGF-1) enhances the protein expression of CFTR. PLoS One 8: e59992.

Ortiz LA, Gambelli F, McBride C, Gaupp D, et al. (2003). Mesenchymal stem cell engraftment in lung is enhanced in response to bleomycin exposure and ameliorates its fibrotic effects. Proc. Natl. Acad. Sci. U. S. A. 100: 8407-8411.

Zhou J (2012). The WJMSCs' Repaired Effects of Oviduct Cell Injury. Doctoral Dissertation of Jinan University. Medical College, Jinan University, Guangzhou. 